\title{
Iterative Frequency-Domain Packet Combining Techniques for UWB Systems with Strong Interference Levels
}

\author{
Mário Marques da Silva - Rui Dinis . \\ Paulo Montezuma
}

(5) Springer Science+Business Media, LLC. 2012

\begin{abstract}
UWB (Ultra Wideband) systems tend to suffer strong interference from signals that occupy a significant part of the transmission band. This is an important constraint, especially when the channel remains fixed for a long period of time. In order to overcome this limitation, this paper considers UWB systems employing Single-Carrier with FrequencyDomain Equalization techniques. We propose the corresponding receiver, which also allows the soft packet combining associated to different Automatic Repeat ReQuest transmission attempts, as a measure to improve the performance through the exploitation of diversity. Our techniques are able to cope with strong interference levels as well as deep fading, even for fixed channels.
\end{abstract}

Keywords ARQ techniques · Soft combining · Single-carrier modulations ·

Interference mitigation - Frequency-domain equalization - Ultra wide band systems

\section{Introduction}

UWB (Ultra Wideband) signals are characterized by bandwidths that can exceed $25 \%$ of the central frequency of the spectrum [1]. Moreover, the low transmit powers combined with the

\author{
M. Marques da Silva $(\varangle) \cdot$ R. Dinis \\ Instituto de Telecomunicações, Lisbon, Portugal \\ e-mail: marques.silva@ieee.org \\ M. Marques da Silva \\ Universidade Autónoma de Lisboa, Lisbon, Portugal \\ M. Marques da Silva \\ Escola Naval/CINAV, Lisbon, Portugal \\ R. Dinis - P. Montezuma \\ DEE-FCT-UNL, Caparica, Portugal \\ P. Montezuma \\ UNINOVA, Caparica, Portugal
}

Published online: 11 June 2012

Springer 